\title{
Priority setting: learning to make tough decisions
}

\section{S R Benatar}

\section{The costs of trying to provide modern medical care for all who could potentially benefit exceed the capacity of most countries}

$\mathrm{P}$ riority setting (also termed resource allocation) for healthcare expenditure is inevitable in all societies, even the most affluent. ${ }^{1}$ New technologies are the dominant driving forces. ${ }^{2}$ Although these advances have been successful both in saving lives and in improving quality of life, the costs of trying to provide such modern medical care for all who could potentially benefit exceed the capacity of most countries.

A very considerable proportion of medical expenditure is generated on treating patients during what turns out to be the last year of life. ${ }^{3}$ It is now increasingly recognised that there are inadequate health returns from such expenditure (both in terms of duration and quality of life) and that there are limits to what medicine should be attempting to achieve. ${ }^{4}$ Consequently there has been a subtle, but largely unacknowledged, shift from a "sanctity of life" approach (in which medical care is continued relentlessly, even when death seems imminent) to "quality of life" considerations. ${ }^{5}$ This is reflected in the wishes of many patients to be spared ongoing heroic measures and to be allowed to die peacefully. Increasingly healthcare professionals are also willing to withdraw treatment when the prognosis seems very poor, or when other goals of health care are considered to be higher priorities. ${ }^{6}$

Additional reasons for interest in priority setting, beyond the appropriateness of aggressive medical care, include the need for more equitable access to care and the desirability of reducing arbitrariness in medical practice through use of practice guidelines informed by the best evidence.

As many values are at stake and there are differing rankings given to these, the debate about priority setting has been heated and acrimonious. In the United Kingdom, strong arguments have been advanced to retain the traditional method of implicit (or covert) rationing. Advantages claimed for implicit rationing are that it is based on trust, and that, because it is more sensitive to the complexity of medical decisions as well as to the personal and cultural preferences of patients, it is the best method at the micro level of individual patient care. Medical practitioners are accustomed to making such decisions at the bedside or the clinic and they value their autonomy to do so. Advocates for implicit rationing see several disadvantages to explicit (overt, reasoned, and accountable) rationing. They have doubts about the assumption that explicit rationing is practical and possible, and believe that explicitness will distress patients and providers. They are also troubled by the administrative complexity of the process, its potential to destabilise the healthcare system, and the lack of responsiveness to rapid changes in knowledge. ${ }^{78}$

Advocates of explicit rationing claim that equal need and equal access (NHS principles), the unavoidable need for triage, and the ability to link triage to waiting lists, are good reasons to undertake rationing on an explicit basis. Moreover it is argued that the process reduces deception (and ignorance) and is less paternalistic. Rationing is considered to be a requirement in a democracy as it encourages politicians and administrators to reflect on their decisions and, in the process, attain deeper insights into the implications of their decisions. It is also claimed that explicit rationing could contribute to the moral development of citizens by encouraging them not to believe that they are entitled to everything that is technically possible in health care, especially when benefits are only marginal. ${ }^{9}$

The requirements for explicit rationing include the use of techniques such as cost effectiveness analysis, systematic reviews of outcomes, scoring systems such as quality adjusted life years (QUALYs), as well as clarity about objectives, information about costs and outcomes, and the ability to measure performance. A recently published cost effectiveness study of four disease modifying treatments for relapsing and remitting multiple sclerosis illustrates the complexity of such technical processes ${ }^{10}$ and highlights the ongoing need to pursue such studies and accumulate additional follow up data. ${ }^{11}$ The role of randomised control trials in providing the best evidence on which to base practice guidelines is gaining prominence as the best way to justify expenditure and to
Although the best possible evidence is not available for all aspects of treatment, a recent text illustrates the usefulness of an evidence based approach to the clinical practice of neurology. ${ }^{13}$

The above techniques place emphasis on such values as effectiveness and efficiency-both important social values, but not the only ones. Moreover, as they are best applied at the level of comparing different treatments for the same disease and it is more difficult to apply them to compare treatments of different diseases, judgement is also required. This raises the thorny question of which values should be emphasised and whose judgement should influence the establishment of priorities and the selection of a basket of essential services/treatments. Increasingly efforts are being made to seek public opinion despite all the complexity this poses. An emphasis on judgement reveals the role of ethics and values. For example, judgement is required to determine whether preference is given to improving the quality of life (for example palliative care) or to extending the duration of life (for example through provision of more intensive care beds). Similarly, a balance needs to be shaped between favouring the good of individuals and giving priority considerations to the common good.

Central to priority setting is the question of justice. Ideas of what distributive justice means and how it can be achieved vary greatly. ${ }^{14}$ Some believe in individual freedom as the most important political value in the pursuit of fairness. Others are more concerned about equity, solidarity, and contractual theories of justice that could contribute to achieving the ideal of a harmonious public life. Perspectives on justice also differ across disciplines. For example, philosophers are interested in complex and disparate theories of distributive justice, while lawyers concentrate on the right to health care, non-discrimination, and the obligations of healthcare practitioners. Political scientists reflect on the fairness of decision making processes. Medical staff tend to be caught up with the evidence base and considerations of best clinical judgement, while economists stress efficiency.

In the absence of a satisfactory theory of justice that can be applied in everyday practice, American scholars Daniels and Sabin have proposed a process for priority setting that they have called "accountability for reasonableness". This framework requires that a fair priority setting process meets four conditions. Firstly, the rationale for decisions must be publicly accessible. Secondly, the decisions to meeting healthcare needs must be contextually relevant to fair minded people. Thirdly, allowance must be made for appeals so that previous decisions can 
be reconsidered in the light of new evidence or arguments. Fourthly, there must be a process of enforcement that facilitates the implementation of the last three conditions. ${ }^{15}$

Priority setting needs to be addressed at several levels with different implications and even different approaches at each level. At national levels the budget for public health services is determined largely by the fiscal policy of governments-hopefully influenced in democratic countries by the "will of the people" and the priority they give to health care. Within regional geographical areas, allocation decisions are potentially influenced by policies of both national and regional governments. For particular diseases or forms of treatment-for example head injuries, psychiatric care, or diseases that are expensive to treat, such as multiple sclerosis-resource allocation decisions are shaped by central, regional, and local health authorities. Landman and Henley have proposed that equity can be preserved to some extent against considerations of effectiveness and efficiency by introducing the principle of non-abandonment at the macro level of resource allocation. This requires that a relevant proportion of the overall health budget be allocated to each speciality in medical practice to ensure that all patients, even those requiring expensive treatments, have at least some opportunity to be treated. ${ }^{16}$ Committees within medical institutions are increasingly used to craft policies regarding eligible groups of patients for expensive therapies and they would need to work within national frameworks that preserve equity. Specific treatment decisions for individual patients are characteristically made by practitioners at the bedside, within the context of institutional frameworks.

The framework proposed by Daniels and Sabin is of potential value at each level. Although this was developed in the context of the United States privately funded healthcare system, Martin and colleagues have shown that it is workable and acceptable within the Canadian publicly funded system. ${ }^{17}$ The centrality of fairness in priority setting became clear in their empirical study of priority setting processes for new technologies in cancer and cardiac care in Ontario. They identified the importance of seeking multiple perspectives on the problem under review, ensuring transparency and honesty in the decision making process, identifying potential conflicts of interest, and achieving consensus. The recent detailed qualitative study by Martin and colleagues of how the framework of accountability for reasonableness can be applied to access to intensive care units (ICUs) for neurosurgery patients provides evidence that such a process improves the fairness of priority setting. ${ }^{18}$

Little is known about how resource allocation decisions are made in many countries, but some places for example The Netherlands, Sweden, New Zealand, the United Kingdom, and the state of Oregon in the United States, have moved to explicit rationing processes. While the specific approaches in each of these places differ, common themes include the importance of well publicised individual cases in bringing priority setting to public attention and the need to consult and involve the public to ensure that their views and values contribute to making judgements. ${ }^{19}$

It is doubtful whether more than a few people know how resources are allocated at national, regional, or institutional levels. Even at the level of major hospitals such decisions seem to be made covertly and within a vacuum. Although many are acutely aware of the challenges at the micro level of the doctor-patient interaction, there is not yet an established culture of trying to make such decisions in a rational and accountable mannereven within increasingly resource constrained academic centres.

In the 1990s neurosurgeons at the University of Cape Town's major teaching hospital (Groote Schuur Hospital, South Africa) faced several challenges under conditions of severe resource constraint. These included a rising number of severe head injuries, reduction in the number of ICU and rehabilitation beds, and in operating theatre facilities for neurosurgery. In order to prevent almost all elective neurosurgery from being eclipsed by trauma surgery it became necessary to formulate a policy to prioritise treatment of severe head injures. ${ }^{20}$

Developing this policy provided several lessons. The first is that development of a protocol allowing for resuscitation and full evaluation of every patient and limiting ongoing aggressive care to those with the best prognosis can be undertaken as a cooperative endeavour between physicians, surgeons, bioethicists, lawyers, and administrators-along the lines recommended by Daniels and Sabin. The second is that with sensitive communication families can successfully be brought into the decision making process for withdrawing aggressive life support care from those with the worst prognoses. The third is that having fashioned a rational policy many difficulties have to be faced in its operationalisation. Last but not least is that caution is needed to ensure that the exercise is not used perversely by fiscally oriented politicians to make further cuts in the budget for the care being rationed.

In conclusion, it needs to be acknowledged that priority setting is a complex process for which there is no simple or technical solution. The process is an exercise in policy learning, a struggle for power with varying implications at each of the levels described above, and is undertaken in different ways in different countries. ${ }^{21}$ Although significant progress is being made towards improving fairness in the use and distribution of health services, much remains to be done.

J Neurol Neurosurg Psychiatry 2003;74:1185-1186

\section{Author's affiliation}

S R Benatar, Department of Medicine, University of Cape Town, Observatory, 7925, Cape, South Africa;

sbenatar@uctgsh 1.uct.ac.za

\section{REFERENCES}

1 Daniels N, Sabin JE. Setting limits fairly: can we learn to share medical resources? Oxford: Oxford University Press, 2002

2 Newhouse JP. Medical care costs: how much welfare loss? Journal of Economic Perspectives 1992;6:3-21.

3 Emanuel EJ, Emanuel LL. The economics of dying: the illusion of cost savings at the end of life. N Engl J Med 1995;330:540-4.

4 Callahan D. False hopes: why Americas quest for perfect health is a recipe for failure. New York: Simon and Schuster, 1998.

5 Singer P. Rethinking life and death: the collapse of our traditional ethics. New York: Oxford University Press, 1994

6 Prendergast TJ, Luce JM. Increasing incidence of withholding and withdrawal of life support from the critically ill. Am J Respir Crit Care Med 1996;155:15-20.

7 Mechanic D. Dilemmas in rationing health care services: the case for implicit rationing BM 1995;310:1655-9.

8 Coast J. The rationing debate: rationing with the NHS should be explicit: the case against. BM 1997:314:1118-22.

9 Doyal L. The rationing debate: rationing within the NHS should be explicit: the case for. BM 1997;314:1114-18.

10 Chilcot J, McCabe C, Tappenden P, et al. Modelling the cost effectiveness of interferon beta and glatiramer acetate in the management of multiple sclerosis. BM 2003;326:522-6.

11 Miller DH. Evaluating disease modifying treatments in multiple sclerosis [Commentary]. BM 2003;326:522-6.

12 Cochrane Collaboration. www.cochrane.org. Accessed 2 May 2003.

13 Benatar M. Analytic neurology: examining the evidence for clinical practice. Boston: Butterworth-Heinemann, 2002.

14 Arthur J, Shaw W. Justice and economic distribution. Englewood Cliffs: Prentice Hall, 1991

15 Daniels N, Sabin J. Limits to health care: fair procedures, democratic deliberation and the legitimacy problem for insurers. Philosophy \& Public Affairs 1997;26(4):303-50.

16 Landman WA, Henley LD. Equitable rationing of highly specialised services for children: a perspective from South Africa. $J$ Med Ethics 1999;25:224-9.

17 Martin DK, Giacomini M, Singer PA Fairness, accountability for reasonableness, and the views of priority setting decision-makers. Health Policy 2002;61:279-90.

18 Martin DK, Singer PA, Bernstein M. Access to ICU beds for neurosurgery patients: a qualitative case study. J Neurol, Neurosurg Psychiatry 2003;74:1299-303.

19 Ham C. Priority setting in health care: learning from international experience. Health Policy 1997;42:49-66.

20 Benatar SR, Fleischer TE, Peter JC, et al. Treatment of head injuries in the public sector in South Africa. South African Medical Journal 2000;90:790-93.

21 Martin DK, Singer PA, Giacomini M, et al. Priority setting for new technology in medicine: qualitative case study. BM 2000;321:1316-18. 


\section{Acetylcholinesterase inhibition in the treatment of hypotension}

\section{R Schondorf}

\section{Pyridostigmine reduces tilt induced hypotension}

A ssuming an upright posture causes translocation of approximately $800 \mathrm{ml}$ of blood from the intrathoracic venous compartment to veins of the buttocks, pelvis, and legs. The bulk of venous pooling occurs within the first 10 seconds and the total pooling is complete within three to five minutes. ${ }^{1}$ The normal compensatory cardiovascular response to this orthostatic stress is a neurogenically mediated increase in heart rate and systemic vascular resistance. Patients with autonomic failure do not increase systemic vascular resistance, and the decrease in cardiac output during standing is also significant. ${ }^{1}$ The resulting cerebral hypoperfusion causes postural lightheadedness, visual blurring, syncope, focal cerebral ischaemia, and even unexplained falls. In these patients orthostatic hypotension may be significantly exacerbated by prolonged recumbency, food or alcohol ingestion, physical exertion, and vasoactive drugs. Patient education directed at avoidance of these stressors, volume expansion with increased sodium and water intake, ${ }^{2}$ and physical countermanoeuvres to activate the skeletal muscle pump to prevent venous pooling constitute the mainstay of treatment of orthostatic hypotension. ${ }^{1}$ This conservative strategy offsets many of the dynamic and rapid changes in blood pressure that occur during normal activities of daily living. Improved cerebral autoregulation in some patients with autonomic failure may also reduce symptoms of orthostatic hypotension.

Drugs that expand the plasma volume (fludrocortisone) or that supplement peripheral $\alpha$ adrenergic activity (midodrine) are the main pharmacological modes of treatment of these patients. However, the efficacy of these drugs is often unpredictable, depending on the interaction between residual autonomic activity, neurohumoral counterregulatory mechanisms, and the pharmacological agent. For example, administration of aldosterone analogues such as fludrocortisone to normal subjects on an adequate salt intake initially causes sodium and water retention leading to weight gain and a rise in blood pressure. However, within a few days, spontaneous diuresis ensues and plasma volume returns to near normal values - a phenomenon known as the aldosterone escape. Some of the counter-regulatory mechanisms implicated in the aldosterone escape include reductions in the secretion of renin-angiotensin, increased secretion of atrial natriuretic factor, and pressure natriuresis. Baseline sympathetic activity is also significantly diminished by fludrocortisone, even when no increase in plasma volume is observed. Acute administration of the $\alpha$ adrenergic agonist midodrine increases blood pressure, total peripheral resistance, and venomotor activity but decreases heart rate, plasma volume, and muscle sympathetic nerve activity. The diminished heart rate and sympathetic activity are likely to reflect acute activation of baroreceptors, whereas the hypovolaemia is probably related to $\alpha$ adrenergic mediation of vascular smooth muscle

Given this level of unpredictability it is interesting that we know very little about the long term value of these commonly used treatments. The longest placebo controlled study of the efficacy of midodrine was only six weeks, ${ }^{3}$ and there is no placebo controlled trial showing long term efficacy of fludrocortisone in autonomic failure. Both of these drugs have potentially deleterious side effects, the most feared being supine hypertension to a level sufficient to cause target organ damage.

From the above discussion it is evident that a drug that would amplify dynamic contraction. residual autonomic function without the escape phenomena described above and without promoting supine hypertension would be very desirable. In this edition of the journal, Singer et al describe the efficacy a potentially ideal therapeutic agent, pyridostigmine, in reducing tilt induced hypotension. ${ }^{5}$ These investigators suggest that the mechanism of action is the potentiation of sympathetic cholinergic ganglionic transmission. Thus sympathetic activity would be amplified during orthostatic stress and would be minimised while supine. One would imagine, therefore, that those patients with preserved ganglionic transmission would be preferentially improved by pyridostigmine. There was, however, no difference in the response of patients with peripheral and central autonomic failure, nor was the response related to the severity of autonomic failure. Whether pyridostigmine would serve to reduce supine hypertension is also unknown as many patients with peripheral autonomic failure have supine hypertension even after complete ganglionic blockade. ${ }^{4}$ Despite these limitations, given the paucity of effective agents for the treatment of orthostatic hypotension, a properly designed multicentre placebo controlled trial of the efficacy of pyridostigmine in the treatment of autonomic failure would be welcomed by those who treat patients with this condition.

J Neurol Neurosurg Psychiatry 2003;74:1187

\section{Authors' affiliations}

R Schondorf, Sir Mortimer B Davis Jewish General Hospital, 3755 Chemin De La Cote Ste Catherine, Montreal, Quebec H3T IE2, Canada; ronald.schondor@@mcgill.ca

\section{REFERENCES}

1 Smit AAJ, Halliwill JR, Low PA, et al. Pathophysiological basis of orthostatic hypotension in autonomic failure. J Physiol (Lond) 1999;519:1-10.

2 Shannon JR, Diedrich A, Biaggioni I, et al. Water drinking as a treatment for orthostatic syndromes. Am J Med 2002;1 12:355-60.

3 Low PA, Gilden JL, Freeman R, et al. Efficacy of midodrine vs placebo in neurogenic orthostatic hypotension - a randomized, double-blind multicenter study. JAMA 1997; 277: 1046-51

4 Shannon JR, Jordan J, Diedrich A, et al. Sympathetically mediated hypertension in autonomic failure. Circulation 2000;101:2710-15.

5 Singer W, Opfer-Gehrking TL, McPhee BR, et al. Acetylcholinesterase inhibition: a novel approach in the treatment of neurogenic orthostatic hypotension. J Neurol Neurosurg Psychiatry 2003;72:1294-8. 